\title{
MANAGEMENT OF MECKEL'S DIVERTICULUM- A RETROSPECTIVE STUDY IN A TERTIARY CARE CENTRE
}

\author{
A. Ramesh'1, R. Rajavelu2, A. Mohammed Imran Khan ${ }^{3}$ \\ ${ }^{1}$ Assistant Professor, Department of General Surgery, Government Vellore Medical College. \\ 2 Professor, Department of General Surgery, Government Vellore Medical College. \\ 3Junior Resident, Department of General Surgery, Government Vellore Medical College.
}

\section{ABSTRACT}

\section{BACKGROUND}

The aim of this study was to review the management of Meckel's diverticulum in patients admitted in Department of General Surgery in Government Vellore Medical College.

\section{MATERIALS AND METHODS}

- This retrospective study included 45 patients (25 male and 20 female), who underwent exploratory laparotomy for the management of symptomatic Meckel's diverticulum and PVID between January 1, 2014 and January 1, 2016.

- $\quad$ The data were analysed by resources from Medical Records Department in Government Vellore Medical College.

- $\quad$ Forty five patients were retrospectively analysed in the study depending on the preoperative and intraoperative findings.

\section{RESULTS}

During 2 years from January 2014 to January 2016,

- 45 (25 male and 20 female) patients were treated with Meckel's diverticulum.

- 3 patients were under 12 years of age and remaining were above 12 years.

- The mean age group was between 8 to 42 years.

- $\quad$ Lowest age of presentation was 7 years and highest age was 42 years.

- Majority presented with intestinal obstruction, which was followed by perforation peritonitis, lower gastrointestinal bleeding/melaena and as incidental findings during laparotomy for others.

- The surgical procedures done include segmental resection of ileum containing Meckel's diverticulum and ileoileal anastomosis, diverticulectomy with wedge resection, ascending colon anastomosis with ileostomy.

\section{CONCLUSION}

Meckel's diverticulum may present as intestinal obstruction, perforation peritonitis and diverticulitis, lower GI bleeding and some of them with gangrenous bowel. Urgent surgical intervention is needed to prevent morbidity as well as mortality. Symptomatic Meckel's diverticulum and PVID is also an important cause of acute abdomen/intestinal obstruction in children and adults and delay in seeking treatment is associated with high mortality.

\section{KEYWORDS}

Meckel's Diverticulum, Diverticulitis, Wedge Resection, Ileostomy, Patent Vitellointestinal Duct.

HOW TO CITE THIS ARTICLE: Ramesh A, Rajavelu R, Khan AMI. Management of Meckel's diverticulum- A retrospective study in a tertiary care centre. J. Evolution Med. Dent. Sci. 2017;6(24):1992-1997, DOI: 10.14260/Jemds/2017/436

\section{BACKGROUND}

Meckel's diverticulum is the most common congenital anomaly of the small intestine and it is caused by an incomplete obliteration of the vitelline duct/omphalomesenteric duct. Meckel's diverticula are mostly asymptomatic, but it may present as intestinal obstruction, diverticulitis, lower gastrointestinal (GI) bleeding and perforation peritonitis.

Meckel's diverticulum occurs in 2\% population and may present at any age and a person with Meckel's diverticulum has a 4 to $6 \%$ lifetime risk of developing a complication.[1]

Financial or Other, Competing Interest: None.

Submission 17-01-2017, Peer Review 07-02-2017,

Acceptance 16-02-2017, Published 23-03-2017.

Corresponding Author:

Dr. A. Ramesh,

Assistant Professor

Department of General Surgery,

Government Vellore Medical College.

E-mail: ramesharunachalam81@gmail.com

DOI: $10.14260 /$ jemds $/ 2017 / 436$

\section{MATERIALS AND METHODS}

- This retrospective study included 45 patients (25 male and 20 female) who underwent exploratory laparotomy for the management of symptomatic Meckel's.

- Diverticulum and PVID between January 1, 2014 and January 1, 2016.

- The data were analysed by resources from Medical Records Department in Government Vellore Medical College.

- $\quad$ Forty five patients were retrospectively analysed in the study depending on the Preoperative and Intraoperative findings.

\section{RESULTS}

During 2 years from January 2014 to January 2016,

- 45 (25 male and 20 female) patients were treated with Meckel's diverticulum.

- 3 patients were under 12 years of age and remaining were above 12 years.

- $\quad$ The mean age group was between 8 to 42 years. 
- $\quad$ Lowest age of presentation was 7 years and highest age was 42 years.

- Majority presented with intestinal obstruction which was followed by perforation peritonitis, lower gastrointestinal bleeding/melaena and as incidental findings during laparotomy for suspected peritonitis.

- The surgical procedures done include segmental resection of ileum containing Meckel's diverticulum and ileoileal anastomosis, wedge resection and ileostomy.

- Findings during laparotomy include:

1. Meckel's diverticulum with ileoileal band.

2. Perforated Meckel's diverticulum.

3. Diverticulitis/bleeding.

4. Meckel's diverticulum with segmental gangrene of ileum.

5. Normal Meckel's diverticulum as incidental finding found during-

1. Exploration for appendicitis- 2 cases.

2. Laparotomy for peritonitis- 1 case.

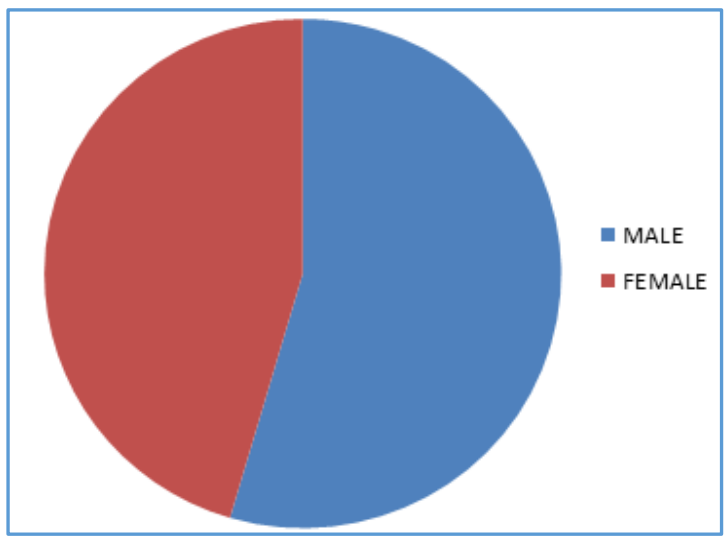

Figure 1. Sex Distribution

\begin{tabular}{|c|c|c|}
\hline Sex & \multicolumn{2}{|c|}{ Frequency } \\
\hline Male & \multicolumn{2}{|c|}{25} \\
\hline Female & \multicolumn{2}{|c|}{20} \\
\hline \multicolumn{3}{|c|}{ Table 1. Sex Distribution } \\
\hline \multicolumn{2}{|c|}{ Pattern } & Frequency \\
\hline \multicolumn{2}{|c|}{ Intestinal Obstruction } & 21 \\
\hline \multicolumn{2}{|c|}{ Perforation Peritonitis } & 9 \\
\hline \multicolumn{2}{|c|}{ Bleeding PR, Melaena } & 7 \\
\hline \multicolumn{2}{|c|}{ Gangrene-Bowel } & 5 \\
\hline \multicolumn{2}{|c|}{ Incidental Finding } & 3 \\
\hline \multicolumn{3}{|c|}{ Table 2. Pattern of Presentation } \\
\hline
\end{tabular}

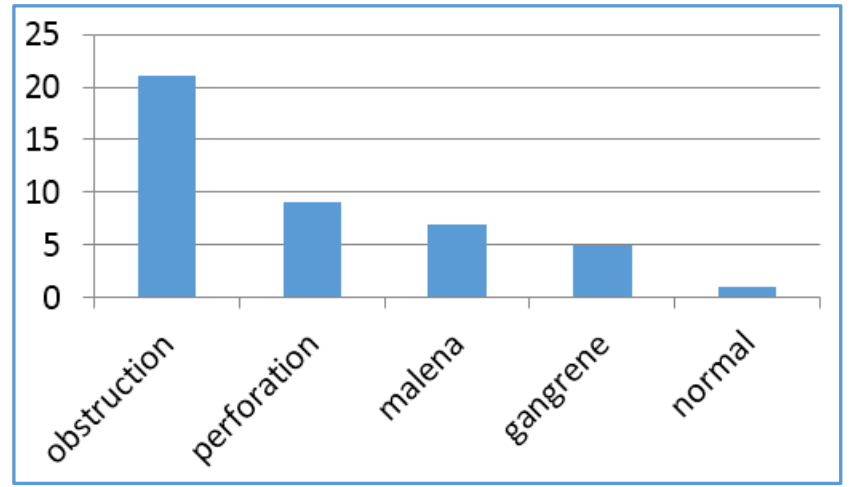

Figure 2. Pattern of Presentation
Segmental Resection with Ileoileal Anastomosis

Diverticulectomy with Wedge Resection

Ileo-ascending Colon Anastomosis with Ileostomy

Table 3. Treatment offered for Meckel's Diverticulum

\section{DISCUSSION}

Meckel's diverticulum is a blind segment of small bowel of about 3-6 cm located in the distal ileum, usually within 60$100 \mathrm{~cm}$ (2 feet) of the ileocecal valve. This blind segment may have a greater lumen diameter than that of the ileum. It runs antimesenterically and has its own blood supply. It is a true diverticulum, consisting of all 3 layers of the bowel wall which are mucosa, submucosa and muscularis propria. It is a remnant of the connection from the yolk sac to the small intestine present during embryonic development.

\section{Clinical significances}

\section{Site for heterotrophic Cell Rests}

As the vitelline duct is made up of pluripotent cell lining, Meckel's diverticulum may harbour abnormal tissues, containing embryonic remnants of other tissue types. Jejunal, duodenal mucosa or Brunner's tissue were each found in $2 \%$ of ectopic cases. Heterotopic rests of gastric mucosa and pancreatic tissue are seen in $60 \%$ and $6 \%$ of cases respectively. Patients with functioning ectopic rests of gastric and pancreatic tissue leads to increased secretion and resulting in epigastric pain. Heterotopic means the displacement of an organ from its normal anatomic location.[2]

\section{Clinical features simulating appendicitis}

Inflammation of this Meckel's diverticulum may present with severe periumbilical pain and may mimic appendicitis. Therefore, during appendectomy, ileum should be checked for the presence of Meckel's diverticulum, if it is found to be present it should be removed along with appendix. It forms an important differential diagnosis for appendicitis. So in case of suspected appendicitis, upon exploration if appendix is found to be normal, survey should be made for the presence of Meckel's diverticulum and diverticulectomy to be done accordingly.

3. Presenting feature as indirect hernia with or without obstruction

It can also be present as an indirect hernia, typically on the right side, where it is known as a "Hernia of Littré". A case report of strangulated umbilical hernia with Meckel's diverticulum has also been published in the literature. [3]

4. Presenting feature as umbilical fistula/hernia/sepsis It can be attached to the umbilical region by the vitelline ligament, with the possibility of vitelline cysts, or even a patent vitelline canal forming a vitelline fistula when the umbilical cord is cut.

Rule of 2s (7) is a useful clinicoanatomical guide for easy remembrance of the features of Meckel's diverticulum.

- $2 \%$ of population.

- 2 feet (proximal to the ileocaecal valve).

- 2 inches in length. 
- 2 types of common ectopic tissue.

- 2 years is the most common age of presentation.

- $\quad$ 2:1 male:female ratio.

However, the exact values for the above criteria range from 0.2-5.

\section{Symptoms}

Majority of people with a Meckel's diverticulum are asymptomatic. An asymptomatic Meckel's diverticulum is called a silent Meckel's diverticulum.[4] Symptoms typically appear before the age of two years.

The most common presenting symptom is painless rectal bleeding such as melaena-like black offensive stools, followed by features of intestinal obstruction, volvulus and intussusception. Occasionally, Meckel's diverticulitis may present with all the features of acute appendicitis. Also, severe pain in the epigastric region is experienced by the patient along with bloating in the epigastric and umbilical regions. At times, the symptoms are so painful that they may cause sleepless nights with acute pain felt in the foregut region, specifically in the epigastric and umbilical regions.

\section{Diagnosis}

Technetium-99m Pertechnetate Scan with a Meckel's Diverticulum.

A technetium-99m (99mTc) pertechnetate scan, also called Meckel scan, is the investigation of choice to diagnose Meckel's diverticula in children. This scan detects gastric mucosa; since approximately $50 \%$ of symptomatic Meckel's diverticula have ectopic gastric or pancreatic cells contained within them,[5] this is displayed as a spot on the scan distant from the stomach itself. In children, this scan is highly accurate and noninvasive, with $95 \%$ specificity and $85 \%$ sensitivity[6]; however, in adults the test is only $9 \%$ specific and $62 \%$ sensitive.

Patients with these misplaced gastric cells may experience peptic ulcers as a consequence. Therefore, other tests such as colonoscopy and screenings for bleeding disorders should be performed, and angiography can assist in determining the location and severity of bleeding. Colonoscopy might be helpful to rule out other sources of bleeding but it is not used as an identification tool.

Angiography might identify brisk bleeding in patients with Meckel's diverticulum.

Ultrasonography could demonstrate omphaloenteric duct remnants or cysts. Computed tomography (CT scan) might be a useful tool to demonstrate a blind ended and inflamed structure in the mid-abdominal cavity, apart from appendix.

In asymptomatic patients, Meckel's diverticulum is often diagnosed as an incidental finding during laparoscopy or laparotomy.

\section{Treatment}

Treatment is surgical, preferably with a laparoscopic approach.[6] Indications for open approach by laparotomy include 1. Patients presenting as emergency with severe peritonitis and sepsis 2. Patients with compromised cardiorespiratory status and risky to subject to general anaesthesia. Advantages of laparoscopic approach include less postoperative pain, early recovery, early return of activity.

In patients with bleeding, strangulation of bowel, bowel perforation or bowel obstruction, treatment involves surgical resection of both the Meckel's diverticulum itself along with the adjacent bowel segment, and this procedure is called a "small bowel resection".[6]

In patients presenting with diverticulum and small bowel gangrene, segmental resection of small bowel with ileoileal anastomosis or ileo-ascending colon anastomosis should be done with proximal protective ileostomy. In cases presenting with extensive gangrenous segments, segmental resection with ileostomy should be planned.

In patients without any of the aforementioned complications, treatment involves surgical resection of the Meckel's diverticulum only, and this procedure is called a simple diverticulectomy.[6]

With regards to asymptomatic Meckel's diverticulum, some recommend that a search for Meckel's diverticulum should be conducted in every case of appendectomy/laparotomy done for acute abdomen, and if found, Meckel's diverticulectomy or resection should be performed to avoid secondary complications arising from it.

\section{Epidemiology}

Meckel's diverticulum occurs in about $2 \%$ of the population. [2] Prevalence in males is 3-5 times higher than in females.[7] Only $2 \%$ of cases are symptomatic, which usually presents among children at the age of 2 .

Most cases of Meckel's diverticulum are diagnosed when complications manifest or incidentally in unrelated conditions such as laparotomy, laparoscopy or contrast study of the small intestine. Classic presentation in adults includes intestinal obstruction and inflammation of the diverticulum (diverticulitis). Painless rectal bleeding most commonly occurs in toddlers.

Detailed knowledge of the pathophysiological properties is essential in dealing with the life-threatening complications of Meckel's diverticulum. [6]

\section{Developmental Anatomy}

During embryonic development, the omphalomesenteric duct (Omphaloenteric duct, vitelline duct or yolk stalk) normally connects the embryonic midgut to the yolk sac ventrally, providing nutrients to the midgut during embryonic development. The vitelline duct narrows progressively and disappears between the 5th and 8th week's gestation.

In Meckel's diverticulum, the proximal part of vitelline duct fails to regress and involute, which remains as a remnant of variable length and location. The solitary diverticulum lies on the antimesenteric border of the ileum (opposite to the mesenteric attachment) and extends into the umbilical cord of the embryo. The left and right vitelline arteries originate from the primitive dorsal aorta, and travel with the omphaloenteric duct. The right becomes the superior mesenteric artery that supplies a terminal branch to the diverticulum, while the left involutes.[6] Having its own blood supply, Meckel's diverticulum is susceptible to obstruction or infection. 


\section{Complications}

The lifetime risk for a person with Meckel's diverticulum to develop certain complications is about $4-6 \%$. Gastrointestinal bleeding, peritonitis or intestinal obstruction may occur in $15-30 \%$ of symptomatic patients. Only $6.4 \%$ of all complications requires surgical treatment; and untreated Meckel's diverticulum has a mortality rate of $2.5-15 \%$.

\section{Pathophysiology of complications \\ Haemorrhage}

Bleeding of the diverticulum is most common in young children, especially in males who are less than 2 years of age. Symptoms may include bright red blood in stools (Haematochezia), weakness, abdominal tenderness or pain, and even anaemia in some cases.

\section{Haemorrhage may be caused by:}

- $\quad$ Ectopic gastric or pancreatic mucosa:

1. Where diverticulum contains embryonic remnants of mucosa of other tissue types.

2. Secretion of gastric acid or alkaline pancreatic juice from the ectopic mucosa leads to ulceration in the adjacent ileal mucosa i.e. peptic or pancreatic ulcer.

3. Pain, bleeding or perforation of the bowel at the diverticulum may result.

4. Mechanical stimulation may also cause erosion and ulceration.

- Gastrointestinal bleeding may be self-limiting but chronic bleeding may lead to iron deficiency anaemia.

The Appearance of Stools may indicate the Nature of the Haemorrhage-

- Tarry Stools- Alteration of blood produced by slow bowel transit due to minor bleeding in upper gastrointestinal tract

- Bright Red Blood Stools- Brisk haemorrhage

- Stools with Blood Streak- Anal fissure

- $\quad$ "Currant Jelly" Stools- Ischaemia of the intestine leads to copious mucus production and may indicate that one part of the bowel invaginates into another intussusception.

\section{Diverticulitis}

Inflammation of the diverticulum can mimic symptoms of appendicitis, i.e., periumbilical tenderness and intermittent crampy abdominal pain. Perforation of the inflamed diverticulum can result in peritonitis. Diverticulitis can also cause adhesions, leading to intestinal obstruction.

\section{Diverticulitis may Result from}

- Association with the mesodiverticular band attaching to the diverticulum tip where torsion has occurred, causing inflammation and ischaemia.

- Peptic ulceration resulting from ectopic gastric mucosa of the diverticulum

- Following perforation by trauma or ingested foreign material e.g. stalk of vegetable, seeds or fish/chicken bone that become lodged in Meckel's diverticulum.
- Luminal obstruction due to tumours, enterolith, foreign body, causing stasis or bacterial infection.

- Association with acute appendicitis.

\section{Intestinal Obstruction}

Symptoms: Vomiting, abdominal pain and severe or complete constipation.

- The vitelline vessels remnant that connects the diverticulum to the umbilicus may form a fibrous or twisting band (volvulus), trapping the small intestine and causing obstruction. Localised periumbilical pain may be experienced in the right lower quadrant (like appendicitis).

- "Incarceration": When a Meckel's diverticulum is constricted in an inguinal hernia, forming a Littré hernia that obstructs the intestine.

- Chronic diverticulitis causing stricture.

- Strangulation of the diverticulum in the obturator foramen.

- Tumours e.g. carcinoma: Direct spread of an adenocarcinoma arising in the diverticulum may lead to obstruction.

- Lithiasis, stones that are formed in Meckel's diverticulum can:

1. Extrude into the terminal ileum, leading to obstruction

2. Induce local inflammation and intussusception.

- The diverticulum itself or tumour within it may cause intussusception. For example, from the ileum to the colon, causing obstruction. Symptoms of this include "currant jelly" stools and a palpable lump in the lower abdomen. This occurs when the diverticulum inverts into the lumen of the ileum, due to either:

1. An active peristaltic mechanism of the diverticulum that attempts to remove irritating factors.

2. A passive process such as the transit of food.

\section{Umbilical Anomalies}

Anomalies between the diverticulum and umbilicus may include the presence of fibrous cord, cyst, fistula or sinus, leading to:

- Infection or excoriation of periumbilical skin, resulting in a discharging sinus

- Recurrent infection and healing of sinus

- Abscess formation in the abdominal wall

- Fibrous cord increases the risk of volvulus formation and internal herniation

\section{Neoplasm}

Tumours in Meckel's diverticulum may cause bleeding, acute abdominal pain, gastrointestinal obstruction, perforation or intussusception.

\section{- Benign Tumours}

1. Leiomyoma.

2. Lipoma.

3. Vascular and neuromuscular hamartoma. 


\section{- Malignant Tumours}

1. Carcinoids: most common, $44 \%$.

2. Mesenchymal tumours: Leiomyosarcoma, peripheral nerve sheath and gastrointestinal stromal tumours, $35 \%$.

3. Adenocarcinoma, $16 \%$.

4. Desmoplastic small round cell tumour.

\section{Other Complications}

- A diverticulum inside a Meckel's diverticulum (Daughter diverticula).

- Stones and phytobezoar (A bezoar of vegetable fibres) in Meckel's diverticulum.

- Vesicodiverticular fistula.

\section{Review of Literature}

Meckel's diverticulum was first described by Fabricius Hildanus in 1598, but the name derives from the German anatomist.

Johann Friedrich Meckel described the embryological and pathological features in 1809. Anomalies related with the total or partial absence of this involution are shown in $2 \%$ of the population and may present at any age. A wide variety of anomalies may occur and are - Meckel's diverticulum, patent vitello-intestinal duct, fibrous band attached to umbilicus to the small gut, cyst and umbilical polyp. ${ }^{8}$ Meckel's diverticulum is the most common congenital abnormality of the small intestine and it is caused by an incomplete obliteration of the omphalomesenteric duct. Although, Meckel's diverticulum generally remains silent but lifethreatening complications may arise making it an important structure for having a detailed knowledge of its anatomical and pathophysiological properties to deal with such complications.

Symptomatic Meckel's diverticulum may present as diverticulitis, intestinal obstruction, lower GI bleeding and perforation peritonitis. ${ }^{7}$ Meckel's diverticula are mostly asymptomatic. Even complicated disease is hardly diagnosed preoperatively and hence met with fatal outcomes, if not timely intervened. Plain radiograph of abdomen in erect position is most useful when intestinal obstruction or perforation of viscus is the concern. ${ }^{7}$ In majority of the cases of acute abdomen in children, USG of abdomen and pelvis can provide sufficient information. Ultrasound scans of abdomen can reveal intussusception, but not the Meckel's diverticulum as the cause of it. Similarly, CT scan of abdomen can provide features of small-bowel obstruction, intussusception, free fluid in cases with peritonitis, free air, etc., but rarely Meckel's as the cause. ${ }^{6}$ Patients with diverticulitis/painless lower GI bleeding/melaena may be evaluated with a radionuclide scan. Scintigraphy ${ }^{1}$ using technetium-99m pertechnetate (Meckel's scan $^{2}$ ) is considered as the method of choice for the preoperative diagnosis of Meckel's diverticulum. The use of this test in children is of high diagnostic accuracy with sensitivity of $85 \%-95 \%$ and specificity of $90 \%-95 \% .{ }^{9}$

Treatment options for the Meckel's diverticulum and PVID are diverticulectomy/wedge resection and anastomosis, segmental resection containing Meckel's and ileoileal anastomosis and can be done by open surgery or laparoscopically. ${ }^{3}$ In recent decade, laparoscopy is being used more frequently for the management of Meckel's diverticulum and is said to be safe, cost-effective and efficient for the diagnosis as well as for definitive treatment of Meckel's diverticulum. The definitive procedure can be done laparoscopically or by laparoscopic assisted method. Compared with conventional laparotomy, it has the advantage of precise operative diagnosis, less traumatic access, fewer intraoperative and postoperative complications and shorter recovery period. During present study, the surgical procedures done for Meckel's diverticulum and PVID in order of frequency were - segmental resection of ileum containing Meckel's diverticulum and ileoileal anastomosis, ${ }^{4}$ diverticulectomy/wedge resection, segmental resection and ileo-ascending anastomosis. ${ }^{5}$

\section{CONCLUSION}

Meckel's diverticulum constitutes the most common benign malformation of the digestive tube and it may present as intestinal obstruction, perforation peritonitis and diverticulitis, lower GI bleeding and some of them with gangrenous bowel. Urgent surgical intervention is needed to prevent morbidity and mortality.

Symptomatic Meckel's diverticulum and PVID is also an important cause of acute abdomen/intestinal obstruction in children and adults. In our study of 45 cases, except for the 3 cases presented incidentally during exploration for appendicitis and peritonitis, the rest of 42 cases presented with acute lower abdominal pain and/or features of intestinal obstruction. All the cases were diagnosed clinically supported by plain X-ray abdomen, ultrasound and CT imaging. Surgical intervention made as early as possible after achieving preoperative stabilisation. Findings during laparotomy include ileoileal band, perforated Meckel's diverticulum, diverticulitis and gangrene. Surgical procedures performed include segmental resection of ileum containing Meckel's diverticulum and ileoileal anastomosis, wedge resection and ileostomy. All cases were postoperatively followed till time of discharge ensuring recovery. To conclude Meckel's diverticulitis is an important cause for acute abdomen in children and adults. Delay in diagnosis or seeking treatment is associated with high risk of mortality. Proper clinical evaluation with appropriate necessary investigations and timely surgical interventions plays a crucial role in saving patient's life.

\section{REFERENCES}

[1] St-Vil D, Brandt ML, Panic S, et al. Meckel's diverticulum in children: a 20-year review. J Pediatr Surg 1991;26(11):1289-92.

[2] Singh S, Pandey A, Ahmed I, et al. Prolapse of bowel via patent vitello intestinal duct-a rare occurrence. Hernia 2010;15(5):567-9.

[3] Tekou H, Akakpo-Numado GK, Gnassingbe K, et al. Meckel's diverticulum in children: eleven cases. Gastroenterol Clin Biol 2007;31(6-7):617-20.

[4] Shalaby RY, Soliman SM, Fawy M, et al. Laparoscopic management of Meckel's diverticulum in children. J Pediatr Surg 2005;40(3):562-7.

[5] SaiPrasad TR, Chui CH, Singaporewalla FR, et al. Meckel's diverticular complications in children: is laparoscopy the order of the day? Pediatr Surg Int 2007;23:141-7. 
[6] Beyrouti MI, Amar BM, Beyrouti R, et al. Complications of Meckel's diverticulum. Report of 42 cases. Tunis Med 2009;87(4):253-6.

[7] El-Hussuna A, Zeb A, Mogensen AM, et al. Perforation of Meckel's diverticulum with intraabdominal haemorrhage and periappendicular inflammation. Ugeskr Laeger 2009;171(50):3676-7.
[8] Sharma RK, Jain VK. Emergency surgery for Meckel's diverticulum. World J Emergency Surg 2008;3:27.

[9] Yamada T, Seiki Y, Ueda $M$, et al. Patent omphalomesenteric duct: a case report and review of Japanese literature. Asia Oceania J Obstet Gynaecol 1989;15(3):229-36 